University of Nebraska - Lincoln

DigitalCommons@University of Nebraska - Lincoln

Stephen Ducharme Publications

Research Papers in Physics and Astronomy

3-26-2007

\title{
Electric energy density of dielectric nanocomposites
}

Jiangyu Li

University of Washington, jjli@uw.edu

L. Zhang

University of Nebraska - Lincoln

Stephen Ducharme

University of Nebraska, sducharme1@unl.edu

Follow this and additional works at: https://digitalcommons.unl.edu/physicsducharme

Part of the Physics Commons

Li, Jiangyu; Zhang, L.; and Ducharme, Stephen, "Electric energy density of dielectric nanocomposites" (2007). Stephen Ducharme Publications. 28.

https://digitalcommons.unl.edu/physicsducharme/28

This Article is brought to you for free and open access by the Research Papers in Physics and Astronomy at DigitalCommons@University of Nebraska - Lincoln. It has been accepted for inclusion in Stephen Ducharme Publications by an authorized administrator of DigitalCommons@University of Nebraska - Lincoln. 


\title{
Electric energy density of dielectric nanocomposites
}

\author{
J. Y. Li $i^{\mathrm{a})}$ \\ Department of Mechanical Engineering, University of Washington, Seattle, Washington 98195-2600 \\ L. Zhang \\ Department of Mechanical Engineering, University of Washington, Seattle, Washington 98195-2600; \\ Department of Physics and Astronomy, University of Nebraska Lincoln, Nebraska; and Nebraska Center for \\ Materials and Nanoscience, University of Nebraska, Lincoln, Nebraska 68588-0111 \\ Stephen Ducharme \\ Department of Physics and Astronomy, University of Nebraska, Lincoln, Nebraska 68588-0111 and \\ Nebraska Center for Materials and Nanoscience, University of Nebraska, Lincoln, Nebraska 68588-0111
}

(Received 6 December 2006; accepted 21 February 2007; published online 26 March 2007)

\begin{abstract}
Dielectric materials with large electric energy density are actively pursued for many applications. The authors analyze the effective permittivity, breakdown strength, and electric energy density of dielectric nanocomposites using an effective medium approximation, modeling the nanocomposite as a three-phase material by the double-inclusion method. The addition of nanoparticles enhances the permittivity but reduces the breakdown strength, making the potential gain in electric energy density small. In addition, the interfacial interaction shifts the "percolation" threshold toward lower volume fraction of nanoparticles. The analysis suggests that the microstructure of nanocomposites must be carefully controlled to maintain high dielectric strength and therefore realize enhanced electric energy density. () 2007 American Institute of Physics. [DOI: 10.1063/1.2716847]
\end{abstract}

Dielectric materials that are capable of storing large electric energy are highly desirable for many electronic and electric systems for energy pulse and power conditioning applications, ${ }^{1-3}$ and have been actively pursued in the past few years. Since the electric energy density in a dielectric material is limited to $\kappa E_{b}^{2} / 2$, where $\kappa$ is the dielectric constant or permittivity of the material and $E_{b}$ is the breakdown strength, both large permittivity and high breakdown strength are required for large electric energy storage. While ceramic materials usually have large permittivity, they are limited by their relative small breakdown strength. On the other hand, polymers usually enjoy higher breakdown strength but suffer from much smaller permittivity. It is thus not surprising that numerous efforts have been made in the past few years to combine the polymers of high breakdown strength with nanoparticles of high permittivity, ${ }^{4-10}$ with the hope to substantially enhance the electric energy density of the resulting nanocomposites. This approach is appealing for two reasons. First of all, there are large interfacial areas in a nanocomposite, which could promote interfacial exchange coupling through a dipolar interface layer and lead to enhanced polarization and polarizability in polymer matrix near the interface. ${ }^{6,11}$ As a result, enhanced permittivity can be expected in the polymer matrix near the interfaces. Secondly, the nanoscale particles also make it possible to reduce the thickness of polymer matrix film to nanoscale, and thus enhance its already high breakdown strength even further by avoiding avalanche effects. ${ }^{12}$ Both effects are beneficial for enhanced electric energy density, but whether they will be sufficient to compensate the reduction in breakdown strength due to the dielectric inhomogeneity in the presence of nanoparticles remains to be seen, and is of tremendous impor-

${ }^{\text {a) }}$ Author to whom correspondence should be addressed. Electronic mail: jjli@u.washington.edu tance to the development of nanocomposite high-energydensity capacitors.

The composite concept is very appealing at first sight, and enormous efforts have been devoted to the development of enhanced dielectric composites. The theoretical understanding or guidance on nanocomposite dielectrics, on the other hand, is rather limited. Although the inclusion of nanoparticles with high dielectric constants increases the average dielectric constant of a composite, they also produce a highly inhomogeneous electric field with local hot spots of increased electric field concentration and reduced dielectric strength, thus reducing the effective breakdown strength of the composite. ${ }^{13-15}$ Since the electric energy density scales quadratically with electric field, this reduction in breakdown strength could be a serious drawback of nanocomposite approach. The main challenge, therefore, becomes how to balance the seemingly contradictory criteria of enhancing dielectric constant while maintaining high dielectric strength. This letter reports our results of a theoretical study of electric energy density in dielectric nanocomposites to provide some insight to practical trade-offs in the design of high-energydensity dielectric composites, with particular attention to the inevitable but often neglected reduction of breakdown strength.

To answer this question, we model a dielectric nanocomposite as a three-phase material, consisting of a polymer matrix (phase 1), a interfacial phase of fixed thickness $l$ (phase 2 ), and nanoparticle fillers (phase 3), schematically shown in Fig. 1. The interfacial phase is between polymer matrix and nanoparticles, and can be viewed as a core-shell type of structure together with nanoparticles. Our previous studies suggest that the interfacial length is governed by exchange constant and permittivity of polymer, ${ }^{11}$ and thus it is reasonable to assume that the interfacial phase has fixed thickness, independent of nanoparticle size. As such, the volume fraction of the interfacial phase is given by 


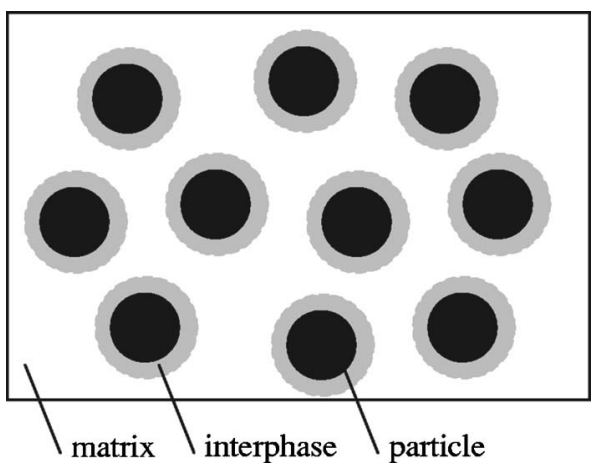

FIG. 1. Schematic diagram of a dielectric nanocomposite consisting of polymer matrix, nanoparticles, and interfacial phase.

$$
f_{2}=\frac{(r+l)^{3}-r^{3}}{r^{3}} f_{3},
$$

where $f_{3}$ is the volume fraction of nanoparticles and $r$ is the nanoparticle radius. From Eq. (1) it is clear that the interfacial fraction $f_{2}$ increases substantially when the nanoparticle size decreases. For this three-phase composite, the effective permittivity $\kappa^{*}$ can be expressed exactly as

$$
\kappa^{*}=\kappa_{1}+f_{2}\left(\kappa_{2}-\kappa_{1}\right) a_{2}+f_{3}\left(\kappa_{3}-\kappa_{1}\right) a_{3},
$$

where $a_{r}$ is the electric field concentration factor for corresponding phase $r$, which relates the average electric field in phase $r$ to that applied at boundary, $E_{0}$,

$$
\left\langle E_{r}\right\rangle=a_{r} E_{0} .
$$

When there is a large contrast in permittivity between polymer matrix and nanoparticles, our previous analysis shows that the effective medium approximation gives reasonable estimation of electric field concentration factor $a_{r} \cdot{ }^{15-17}$ For the core-shell type of microstructure that we are considering, it can be shown from the double-inclusion method that ${ }^{18-20}$

$$
a_{r}=1-s\left[\left(\kappa_{r}-\kappa^{*}\right)^{-1} \kappa^{*}+s\right]^{-1}, \quad r=2,3,
$$

where $s$ is the component of the dielectric Eshelby tensor that is related to the depolarization factor. For spherical particles we are considering $s=1 / 3$. Notice that $\kappa^{*}$ appears on both sides of Eq. (2), necessitating a numerical solution, in general. When $a_{2}$ and $a_{3}$ are determined from Eq. (4), the electric field concentration factor $a_{1}$ can then be determined from the normalization condition $\sum_{r=1}^{3} f_{r} a_{r}=1$.

To demonstrate the influence of nanoparticles, we calculated the effective permittivity of a nanocomposite as shown in Fig. 2, with $\kappa_{3} / \kappa_{1}=1000$ corresponding to typical ratio of permittivity for ceramic and polymer, $\kappa_{2}=\left(\kappa_{1}+\kappa_{3}\right) / 2$, and $l / r=0.1$, corresponding to particle size around $100 \mathrm{~nm}$ for typical exchange length of a few nanometers. ${ }^{11}$ While the exact nature of the interfacial phase varies from system to system, we believe it will not change the qualitative characteristics significantly. In particular, chemical reactivity with the nanoparticles is not a significant issue with modern fluoropolymers, an excellent candidate for matrix material of dielectric nanocomposites. The dashed line in Fig. 2 ignores the interfacial effect, while solid line accounts for that by three-phase model. It is clear that the effective medium approximation is able to capture the large increase in the effective permittivity beyond a threshold in volume fraction that is often observed in experiments, similar to insulator-

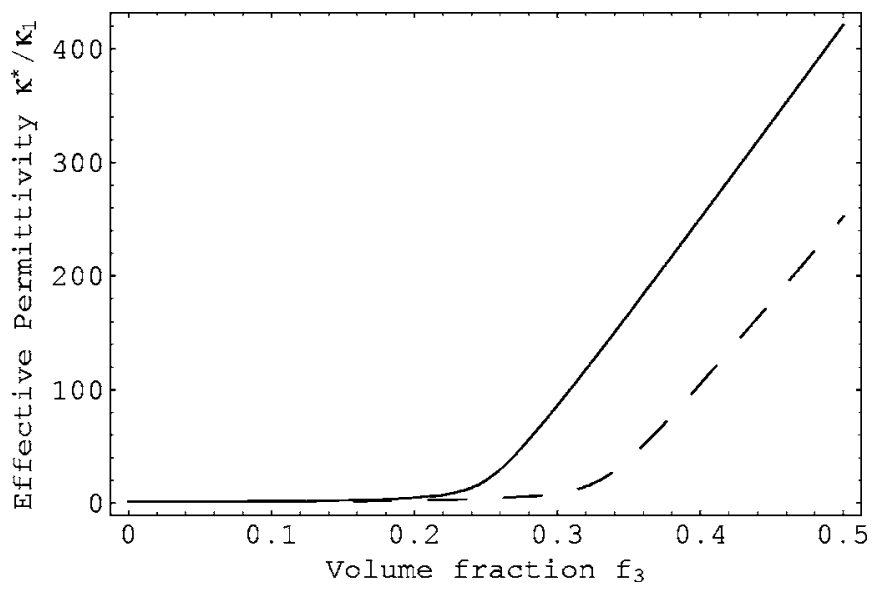

FIG. 2. Normalized effective permittivity of nanocomposite as function of volume fraction of nanoparticles; the dashed line ignores the interfacial effect, while the solid line takes into account the interphase.

conductor percolation transition. In addition, the interfacial exchange coupling shifts the transition threshold toward lower volume fraction, and higher dielectric constant can be expected at the same volume of nanoparticles if the effect of interphase is considered. In other words, nanoparticles can indeed lead to higher dielectric constant in composites compared to microscale particles.

With the addition of nanoparticles of larger permittivity, the average electric field in polymer matrix, $\left\langle E_{1}\right\rangle$, will be enhanced compared to that applied at boundary. This leads to reduction in breakdown strength and a breakdown criterion based on average field,

$$
\left\langle E_{1}\right\rangle=a_{1} E_{0} \leqslant E_{b}
$$

where $E_{b}$ is the breakdown strength of polymer. However, this criterion fails to account for large field fluctuations in the polymer matrix and therefore seriously overestimates the breakdown strength of composites, as we demonstrated in our previous studies. ${ }^{15}$ When the field fluctuation is taken into account, a more reasonable criterion on composite breakdown strength can be proposed,

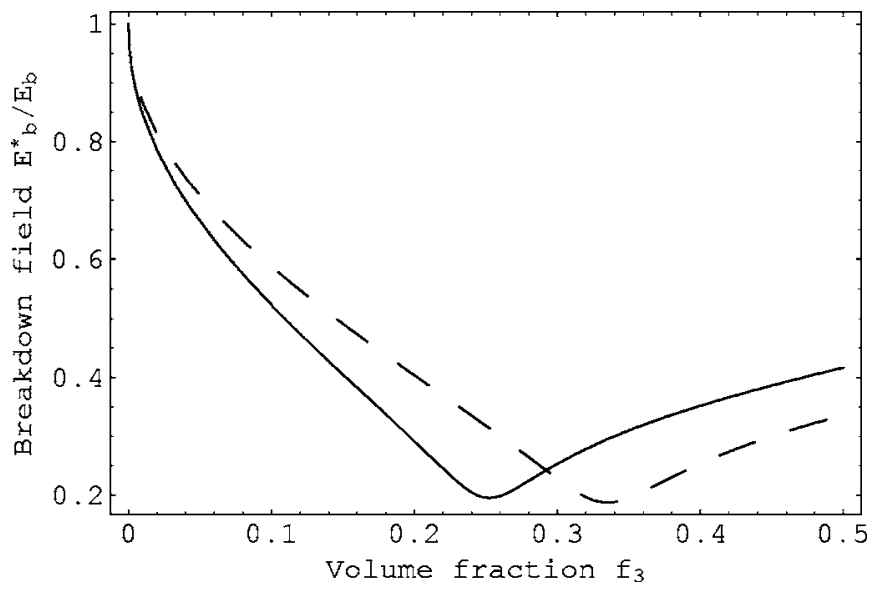

FIG. 3. Normalized breakdown strength of nanocomposite as a function of volume fraction of nanoparticles; the dashed line ignores the interfacial effect, while the solid line takes into account the interphase.

AIP license or copyright, see http://apl.aip.org/apl/copyright.jsp 


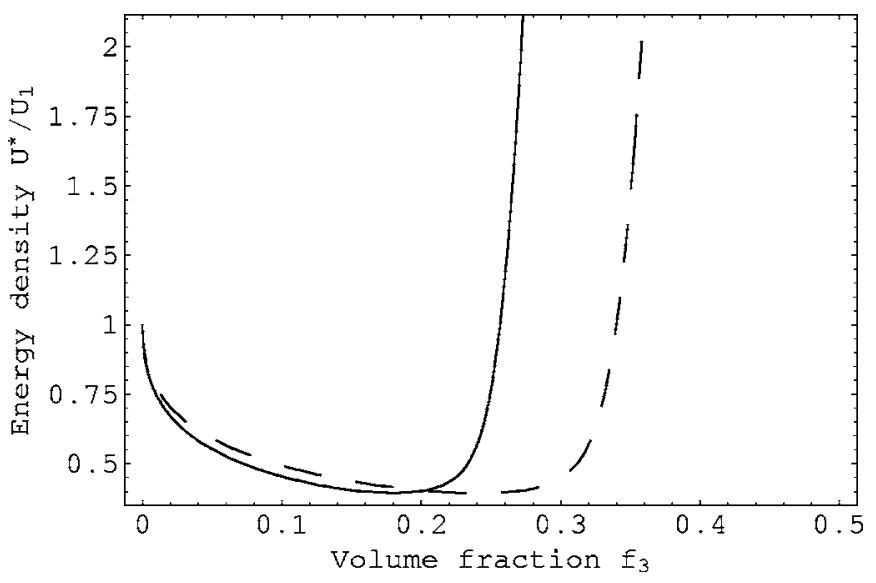

FIG. 4. Normalized electric energy density of nanocomposite as function of volume fraction of nanoparticles; the dashed line ignores the interfacial effect while the solid line takes into account the interphase.

$$
\left\langle E_{1}\right\rangle+\sqrt{\left\langle E_{1}^{2}\right\rangle-\left\langle E_{1}\right\rangle^{2}} \leqslant E_{b},
$$

which agrees with experimental data well. ${ }^{15}$ Thus it is necessary to determine the second order moment of electric field in polymer matrix, which can be determined exactly as ${ }^{15,21}$

$$
\left\langle E_{1}^{2}\right\rangle=\frac{1}{f_{1}} \frac{\delta \kappa^{*}}{\delta \kappa_{1}} E_{0}^{2}
$$

Combining Eqs. (2), (7), and (6) thus allows us to determine the breakdown strength of composites. It is worthwhile to point out that this criterion only considers the field fluctuation in the polymer matrix due to the addition of nanoparticles, and ignore the introduction of defects that could reduce breakdown strength even further. As such, the results can be viewed as an upper bound on the breakdown strength of the composite. The calculated breakdown strength as a function of nanoparticle volume fraction is given in Fig. 3 using the same parameters as before. It is observed that the breakdown strength decreases rapidly with the increase of nanoparticle volume fraction until the percolation threshold is reached. Beyond the percolation transition, the breakdown strength rebounds because the field fluctuation is reduced as nanoparticle fraction increases. However, caution must be excised in drawing any conclusion on breakdown strength beyond the percolation transition, because the increase of other defects is likely to reduce the breakdown strength even further. It is also noted that the interfacial interaction increases the effective dielectric constant but decreases the breakdown strength, and thus gain in electric energy density could be limited. Indeed, as shown in Fig. 4, the difference in electric energy density below percolation transition, with or without an interfacial phase, is rather smaller and the net energy density is smaller than that of pure polymer matrix. Beyond percolation transition, energy density can rise rapidly, but the estimate on breakdown strength is less reliable, making it inconclusive. In any case, the microstructure of nanocomposite must be carefully controlled to expect any gain in electric energy density near the percolation transition.

Finally, we calculate the energy density of the nanocomposite as a function of volume fraction and radius of nanoparticles, as shown in Fig. 5, to demonstrate the size effect in

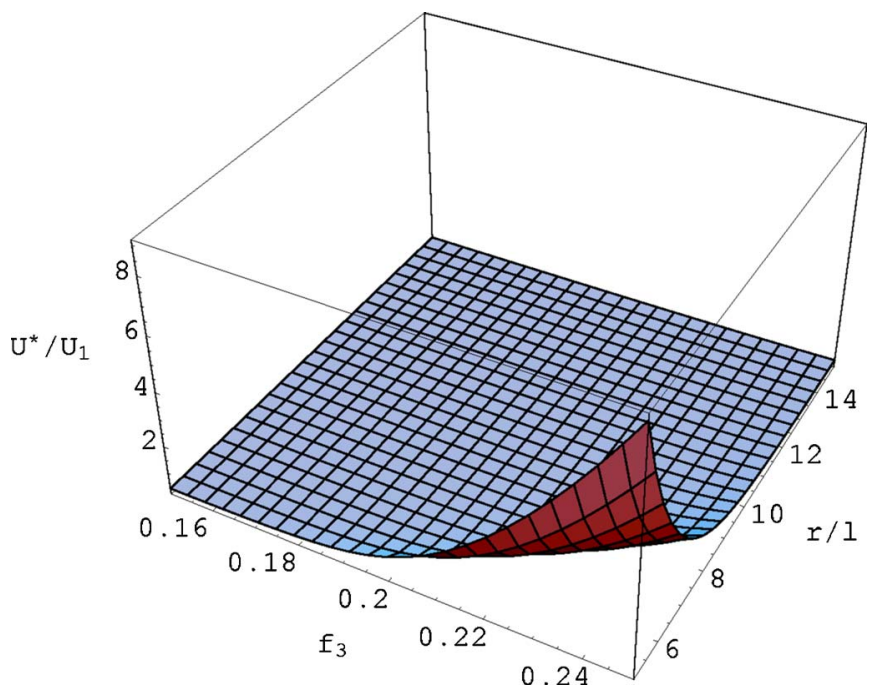

FIG. 5. Normalized electric energy density of nanocomposite as function of volume fraction and size of nanoparticles.

the nanocomposites. It appears that the interfacial exchange interaction starts to be effective when $r / l$ approaches 10, resulting in a rapid increase in energy density as the nanoparticle size is reduced. For a typical exchange length of a few nanometers, it suggests that the particles size should be around $100 \mathrm{~nm}$ or smaller to take advantage of the exchange coupling.

This work is supported by the Office of Naval Research, University of Washington Royalty Research Fund, Bryan T. McMinn Endowed Research Professorship in Mechanical Engineering, and Nebraska Research Initiative.

${ }^{1}$ W. J. Sarjeant, J. Zirnheld, and F. W. MacDougall, IEEE Trans. Plasma Sci. 26, 1368 (1998).

${ }^{2}$ M. Rabuffi and G. Picci, IEEE Trans. Plasma Sci. 30, 1939 (2002).

${ }^{3}$ B. J. Chu, X. Zhou, K. L. Ren, B. Neese, M. R. Lin, Q. Wang, F. Bauer, and Q. M. Zhang, Science 313, 334 (2006).

${ }^{4}$ D. K. Dasgupta and K. Doughty, Thin Solid Films 158, 93 (1988).

${ }^{5}$ Y. Bai, Z. Y. Cheng, V. Bharti, H. S. Xu, and Q. M. Zhang, Appl. Phys. Lett. 76, 3804 (2000).

${ }^{6}$ Q. M. Zhang, H. F. Li, M. Poh, F. Xia, Z. Y. Cheng, H. S. Xu, and C. Huang, Nature (London) 419, 284 (2002).

${ }^{7}$ Y. Rao, S. Ogitani, P. Kohl, and C. P. Wong, J. Appl. Polym. Sci. 83, 1084 (2002).

${ }^{8}$ Z. M. Dang, Y. H. Lin, and C. W. Nan, Adv. Mater. (Weinheim, Ger.) 15, 1625 (2003).

${ }^{9}$ C. Huang and Q. M. Zhang, Adv. Funct. Mater. 14, 501 (2004).

${ }^{10}$ C. Huang, Q. M. Zhang, J. Y. Li, and M. Rabeony, Appl. Phys. Lett. 87, 182901 (2005).

${ }^{11}$ J. Y. Li, Phys. Rev. Lett. 90, 217601 (2003).

${ }^{12}$ A. V. Bune, V. M. Fridkin, S. Ducharme, L. M. Blinov, S. P. Palto, A. V. Sorokin, S. G. Yudin, and A. Zlatkin, Nature (London) 391, 874 (1998).

${ }^{13}$ F. Peruani, G. Solovey, I. M. Irurzun, E. E. Mola, A. Marzocca, and J. L. Vicente, Phys. Rev. E 67, 066121 (2003).

${ }^{14}$ J. P. Calame, J. Appl. Phys. 94, 5945 (2003).

${ }^{15}$ J. Y. Li, C. Huang, and Q. M. Zhang, Appl. Phys. Lett. 84, 3124 (2004).

${ }^{16}$ D. J. Bergman and K. J. Dunn, Phys. Rev. B 45, 13262 (1992).

${ }^{17}$ C. W. Nan, Prog. Mater. Sci. 37, 1 (1993).

${ }^{18}$ M. Hori and S. Nematnasser, Mech. Mater. 14, 189 (1993).

${ }^{19}$ M. L. Dunn and H. Ledbetter, ASME Trans. J. Appl. Mech. 62, 1023 (1995).

${ }^{20}$ J. Y. Li, Int. J. Solids Struct. 37, 5579 (2000).

${ }^{21}$ J. Y. Li and M. L. Dunn, Int. J. Eng. Sci. 37, 665 (1999). 